\title{
Optimal timing of price change with strategic customers under demand uncertainty: A real option approach
}

\author{
Lee, Y.. ${ }^{\text {, Lee, J.P. }}{ }^{\mathrm{b}}$, Kim, S. ${ }^{\mathrm{b}, *}$ \\ ${ }^{a}$ Department of Mechanical Engineering, University of Texas at San Antonio, San Antonio, United States of America (USA) \\ ${ }^{b}$ College of Business Administration, Hongik University, Seoul, South Korea
}

\section{A B S T R A C T}

This paper proposes a model to determine the optimal markdown timing for a company with strategic customer purchasing behaviour. Since strategic customers are aware of potential markdown under the posted pricing scheme, they may choose to wait longer to maximise their utilisation instead of buying a product and fulfilling an instant surplus. On the other hand, the seller can delay the markdown decision until it is proved to be profitable and hence has an option to determine the timing. In estimating the value of the markdown decision, the seller's option needs to be estimated. However, the value of the option is hard to be captured by the conventional net present value analysis. Under market uncertainty where potential customer demand evolves over time, the seller's revenue function is in the form of a stochastic dynamic programming model. Applying a real option approach, we investigate the optimal price path and propose the optimal markdown threshold. Given the markdown costs incurred, we find that the optimal discount timing for the firm is determined by a threshold policy. Furthermore, our results show that if future market becomes more uncertain, the seller needs to wait longer or delay the markdown decision. In addition, the optimal threshold of the markdown decreases exponentially in a declining market, which explains the early markdown policy of some consumer product companies.
\end{abstract}

\section{ARTICLE INFO}

Keywords:

Strategic customers;

Price change;

Posted pricing;

Markdown;

Demand uncertainty;

Real option

*Corresponding author:

sbkim@hongik.ac.kr

(Kim, S.)

Article history:

Received 28 February 2019

Revised 10 September 2019

Accepted 12 September 2019

\section{References}

[1] Albey, E., Norouzi, A., Kempf, K.G., Uzsoy, R. (2015). Demand modeling with forecast evolution: An application to production planning, IEEE Transactions on Semiconductor Manufacturing, Vol. 28, No. 3, 374-384, doi: 10.1109/ TSM.2015.2453792.

[2] Arnold, M.A., Lippman, S.A. (2001). Analytics of search with posted prices, Economic Theory, Vol. 17, No. 2, 447466, doi: $10.1007 / \mathrm{pl00004113}$.

[3] Aviv, Y., Pazgal, A. (2008). Optimal pricing of seasonal products in the presence of forward-looking consumers, Manufacturing \& Service Operations Management, Vol. 10, No. 3, 339-359, doi: 10.1287/msom.1070.0183.

[4] Cachon, G.P., Swinney, R. (2009). Purchasing, pricing, and quick response in the presence of strategic consumers, Management Science, Vol. 55, No. 3, 497-511, doi: 10.1287/mnsc.1080.0948.

[5] Chen, Y., Farias, V.F. (2018). Robust dynamic pricing with strategic customers, Mathematics of Operations Research, Vol. 43, No. 4, 1119-1142, doi: 10.1287/moor.2017.0897.

[6] Chen, Y., Farias, V.F., Trichakis, N. (2019). On the efficacy of static prices for revenue management in the face of strategic customers, Management Science, doi: 10.1287/mnsc.2018.3203.

[7] Dasu, S., Tong, C. (2010). Dynamic pricing when consumers are strategic: Analysis of posted and contingent pricing schemes, European Journal of Operational Research, Vol. 204, No. 3, 662-671, doi: 10.1016/j.ejor.2009. $\underline{11.018}$

[8] Dixit, A.K., Pindyck, R.S. (1994). Investment under uncertainty, Princeton University Press, Princeton, New Jersey, USA. 
[9] Dye, R. (2000). The buzz on buzz, Harvard Business Review, Vol. 78, 139-146.

[10] Gallego, G., Sahin, O. (2006). Inter-temporal valuations, product design and revenue management, Airline Group of the International Federation of Operational Research Societies (AGIFORS).

[11] Gallego, G., Van Ryzin, G. (1994). Optimal dynamic pricing of inventories with stochastic demand over finite horizons, Management Science, Vol. 40, No. 8, 999-1020, doi: 10.1287/mnsc.40.8.999.

[12] Harrison, J.M. (2013). Brownian models of performance and control, Cambridge University Press, Cambridge, United Kingdom, doi: $10.1017 /$ CBO9781139087698.

[13] Kim, S., Huh, W.T., Dasu, S. (2015). Pre-announced posted pricing scheme: Existence and uniqueness of equilibrium bidding strategy, Operations Research Letters, Vol. 43, No. 2, 151-160, doi: 10.1016/j.orl.2014.12.010.

[14] Liu, M., Bi, W., Chen, X., Li, G. (2014). Dynamic pricing of fashion-like multiproducts with customers' reference effect and limited memory, Mathematical Problems in Engineering, Vol. 2014, Article ID 157865, 10 pages, doi: $10.1155 / 2014 / 157865$.

[15] Liu, Q., Van Ryzin, G.J. (2008). Strategic capacity rationing to induce early purchases, Management Science, Vol. 54, No. 6, 1115-1131, doi: $10.1287 /$ mnsc. 1070.0832.

[16] McAfee, R.P., Te Velde, V. (2007). Dynamic pricing in the airline industry, In: Hendershott T.J. (ed.), Handbook of Economics and Information Systems, Vol. 1, Elsevier Science, New York, USA, doi: 10.1016/S1574-0145(06) 01011-7.

[17] McDonald, R., Siegel, D. (1986). The value of waiting to invest, The Quarterly Journal of Economics, Vol. 101, No. 4, 707-727, doi: $10.2307 / 1884175$.

[18] Özer, Ö., Zheng, Y. (2015). Markdown or everyday low price? The role of behavioral motives, Management Science, Vol. 62, No. 2, 326-346, doi: 10.1287/mnsc.2014.2147.

[19] Pindyck, R. (1990). Irreversibility, uncertainty, and investment, Working Paper No. 3307, National Bureau of Economic Research, Cambridge, USA, doi: 10.3386/w3307.

[20] Shen, Z.-J.M., Su, X. (2007). Customer behaviour modelling in revenue management and auctions: A review and new research opportunities, Production and Operations Management, Vol. 16, No. 6, 713-728, doi: 10.1111/ j.1937-5956.2007.tb00291.x.

[21] Smith, S.A., Agrawal, N. (2017). Optimal markdown pricing and inventory allocation for retail chains with inventory dependent demand, Manufacturing \& Service Operations Management, Vol. 19, No. 2, 290-304, doi: $10.1287 / \mathrm{msom} .2016 .0609$.

[22] Stock, A., Balachander, S. (2005). The making of a "hot product": A signalling explanation of marketers' scarcity strategy, Management Science, Vol. 51, No. 8, 1181-1192, doi: 10.1287/mnsc. 1050.0381 .

[23] Su, X. (2007). Intertemporal pricing with strategic customer behaviour, Management Science, Vol. 53, No. 5, 726741, doi: $10.1287 / \mathrm{mnsc} .1060 .0667$.

[24] Talluri, K.T., Van Ryzin, G.J. (2006). The theory and practice of revenue management, Springer-Verlag, New York, USA. 


\section{APEM}

Advances in Production Engineering \& Management Letnik 14 | Številka 3 | September 2019 | Strani 379-390 https://doi.org/10.14743/apem2019.3.335
ISSN 1854-6250

Spletna stran: apem-journal.org Izvirni znanstveni članek

\title{
Optimalen čas spremembe cene pri strateških kupcih v pogojih negotovega povpraševanja: Pristop resničnih možnosti
}

\author{
Lee, Y. ${ }^{a}$, Lee, J.P. ${ }^{b}$, Kim, S. ${ }^{b,}$ \\ ${ }^{a}$ Department of Mechanical Engineering, University of Texas at San Antonio, San Antonio, United States of America (USA) \\ ${ }^{\mathrm{b}}$ College of Business Administration, Hongik University, Seoul, South Korea
}

\section{POVZETEK}

Ta članek predlaga model za določitev optimalnega znižanja cene za podjetje s strateškim nakupnim vedenjem kupcev. Ker se kupci zavedajo potencialnih znižanj v okviru sheme objavljenih cen, se lahko odločijo, da bodo čakali dlje in koristili svoje zaloge, namesto da bi kupili izdelek in dosegli takojšen presežek. Po drugi strani lahko prodajalec odloži odločitev o znižanju cene, dokler se ne izkaže, da je donosna, in ima torej možnost določiti čas. Pri ocenjevanju vrednosti znižanja cene je treba oceniti možnosti prodajalca. Vendar pa je vrednost znižanja cene težko določiti s konvencionalno analizo sedanje neto vrednosti. V tržni negotovosti, kjer se potencialno povpraševanje kupcev spreminja s časom, je prodajalčev prihodek lahko izražen kot funkcija v obliki stohastičnega modela dinamičnega programiranja. Z realističnim pristopom raziskujemo optimalno časovno spreminjanje cene in predlagamo optimalni prag znižanja cene. Glede na nastale stroške znižanja cene ugotavljamo, da je optimalni trenutek za znižanje cene za podjetje določen s pragovno politiko. Poleg tega naši rezultati kažejo, da bo moral prodajalec, če bo prihodnji trg bolj negotov, počakati dlje ali odložiti odločitev o znižanju cene. Poleg tega se optimalni prag znižanja cene na padajočem trgu eksponentno znižuje, kar pojasnjuje politiko zgodnjega znižanja cene nekaterih podjetij za potrošniške izdelke.

\section{PODATKI O ČLANKU}

Ključne besede:

Strateški kupci;

Sprememba cene;

Objavljene cene;

Znižanje cene;

Negotovost povpraševanja;

Resnična možnost

*Kontaktna oseba:

sbkim@hongik.ac.kr

(Kim, S.)

Zgodovina članka:

Prejet 28. februarja 2019

Popravljen 10. septembra 2019

Sprejet 12. septembra 2019 